\title{
FACTORES DEMOGRÁFICOS ASOCIADOS CON EL CONOCIMIENTO SOBRE SALUD EN UNA MUESTRA DE MUJERES DE LA CIUDAD DE LIMA (PERÚ) EN 2008: RESULTADOS DEL CUESTIONARIO SAHLSA-50
}

\author{
Demographic factors associated with health literacy \\ in a sample of females residing in Lima, Peru in \\ 2008: results firom the SAHILSA-50 test \\ Carlos Vílchez-Román, MSc. *, Rocío Quiliano-Terreros**, Rocío Canales-Negrón***, \\ Richard Chiroque-Solano $* * * *$ \\ Recibido: abril 7/09 - Aceptado: agosto 18/09
}

\section{RESUMEN}

Objetivo: identificar el porcentaje de sujetos con un adecuado conocimiento sobre la salud y determinar las variables demográficas que predicen un apropiado conocimiento de la misma.

Metodología: estudio de cohorte transversal en el que se aplicó la prueba SAHLSA-50 en una muestra de 49 mujeres en edad fértil, usuarias del servicio ginecológico del Hospital Dos de Mayo de la ciudad de Lima (Perú). Para someter a prueba la hipótesis se empleó el coeficiente de correlación de Pearson y el análisis de regresión lineal.

Resultados: de acuerdo con lo obtenido, el rango de valores del puntaje de la prueba SAHLSA estuvo entre 22 y 50 puntos con un promedio de $37 \pm 7,15$ puntos. Dichos resultados se vieron reflejados en $43 \%$ de

\footnotetext{
* Magíster. Organización por la Defensa de la Libertad Sexual (ORDELISE). Calle: Javier Luna Pizarro 132, Urbanización La Colonial, Callao (Perú). Teléfono: 511-5616323. Fax: 511-4378289. Correo electrónico: cvilchez@ordelise.org

** Bachiller. Universidad Nacional Mayor de San Marcos (UNMSM), Lima (Perú). Correo electrónico: rocio.terreros@gmail.com

*** Bachiller. Pontificia Universidad Católica del Perú (PUCP), Lima (Perú). Correo electrónico: rocio.canales@pucp.edu.pe

**** Licenciado. Colegio de educación secundaria Altair, Lima (Perú). Correo electrónico: richardchiroque@gmail.com
}

las encuestadas, las cuales consiguieron un puntaje inferior al promedio en la prueba de conocimiento sobre salud ( $\mathrm{n}=21)$. Igualmente, se encontró que la escolaridad es predictora del nivel de conocimiento sobre la salud $(\mathrm{p}<0,05)$.

Conclusiones: los resultados muestran que las pacientes con menor grado de instrucción podrían tener pocas oportunidades de entender los mensajes escritos que provengan de sus servicios de salud.

Palabras clave: conocimiento sobre la salud, mujeres, salud sexual y reproductiva, comunicación en salud.

\section{SUMMARY}

Objective: the objectives were to identify the percentage of subjects having appropriate health literacy and determine the demographic variables predicting appropriate health literacy in females. Methodology: this was a cross-sectional study in which the short assessment of health literacy for Spanish adults (SAHLSA-50) test was given to a sample of 49 reproductive-aged females who were attending the gynaecological service at Dos de Mayo Hospital in Lima, Peru. Linear regression analysis 
and Pearson's coefficient of correlation were used to test the hypothesis.

Results: SAHLSA test scores ranged from 22 to 50 points, averaging $37+7.15$ points; $43 \%$ of the females surveyed obtained a score lower than the average on the health literacy test $(n=21)$. It was found that the level of schooling was a predictor for health literacy level $(\mathrm{p}<0.05)$.

Conclusions: these findings showed that patients who have had lower levels of schooling would have little opportunity of understanding written messages/instructions issued by local or state health services.

Key words: health literacy, female, sexual and reproductive health, health promotion.

\section{INTRODUCCIÓN}

Uno de los indicadores del desarrollo alcanzado por una sociedad es el nivel de alfabetismo; por ello, los gobiernos toman medidas de política educativa y cultural que permitan su incremento. ${ }^{1}$ Según lo informado por el Instituto Nacional de Estadística e Informática de Perú (INEI), entre los años 2002 y 2005, el 12\% de la población mayor de 15 años declaró ser analfabeta, lo que en contraste, equivale a un $88 \%$ de personas que saben leer y escribir. Sin embargo, cuando los estudiantes responden pruebas de comprensión lectora para saber si realmente entienden lo que leen, la cifra del $88 \%$ desciende a menos de $10 \% .^{2-3}$

En ese sentido, dentro de las medidas de alfabetización existe un indicador muy importante que permite saber si las personas tienen la capacidad de entender el material relacionado con el cuidado de la salud (folletos de difusión, formularios y solicitudes de atención médica, instrucciones para tomar pastillas, recetas médicas, etc.): el conocimiento sobre la salud. ${ }^{4}$ Selden y colaboradores lo definen como la capacidad que tienen las personas para obtener, procesar y entender la información básica sobre la salud y los servicios necesarios a fin de tomar decisiones adecuadas sobre el cuidado de ésta. ${ }^{5}$
Llevar a cabo esta medición es importante porque los estudios previos hechos en Perú han revelado que los pacientes potenciales de los servicios de salud conocen poco sobre el tema. ${ }^{6-9}$ A su vez, otros estudios han demostrado que el alto conocimiento sobre la salud está asociado con un mejor cuidado, menores probabilidades de ser internado en el hospital, un menor riesgo de infección con gonorrea y de desarrollo de cáncer cervical, un mejor control de la diabetes, así como el adecuado cuidado de otras enfermedades. ${ }^{10,11}$ Además, un nivel alto de conocimiento sobre la salud permite una mayor comprensión sobre la prevención de las infecciones de transmisión sexual ${ }^{12-14}$ mientras que las personas con un nivel bajo de conocimiento sobre la salud tendrán más dificultades para usar los servicios de los sistemas de salud ${ }^{15}$ y sólo harán uso de ellos cuando estén enfermas. ${ }^{16-17}$

Como se puede deducir, cuanto mayor sea la población con un pobre conocimiento sobre la salud, mayor será la cantidad de personas que demanden los servicios de este sistema, sea público o privado. Todo esto implica que el Estado deba asignar más recursos al presupuesto destinado para atender los programas de salud pública. En síntesis, un bajo nivel de conocimiento sobre la salud genera mayores gastos para el aparato estatal.

Sin embargo, existe controversia acerca de los factores asociados con el conocimiento sobre la salud, en especial en cuanto a la edad, el sexo, el estado civil y los años de escolaridad. ${ }^{18,19}$

Un factor a controlar es el idioma en que se aplica la prueba de conocimiento y el idioma materno. Por ejemplo, en pacientes de origen latino, se encontró que quienes decían tener un manejo fluido del idioma inglés, al contestar pruebas más específicas, su desempeño estuvo por debajo de lo esperado. Además, dicho estudio reveló que en ocasiones existe una marcada diferencia entre la percepción que tienen los médicos y las enfermeras sobre el manejo del idioma por parte de sus pacientes y el desempeño real al leer información relacionada con el cuidado de la salud. ${ }^{20}$ 
Para medir este conocimiento, los investigadores han desarrollado pruebas estándar que permiten evaluar el reconocimiento de palabras y la asociación conceptual de términos vinculados con el cuidado de la salud. Dichas pruebas han sido probadas y validadas en pacientes anglosajones. Por ejemplo, dos cuestionarios muy conocidos, la Prueba de Conocimiento Funcional sobre Salud en Adultos (TOFHLA, por sus siglas en inglés) y el Estimado Rápido de Idioma Adulto en Medicina (REALM, por sus siglas en inglés), son muy empleados por los investigadores norteamericanos. ${ }^{18,19,21}$

Si bien es cierto que estas pruebas cuentan con versiones en español, han sido desarrolladas pensando en pacientes de lengua inglesa ${ }^{9,15,18,21-23}$ y se ha informado acerca de problemas de interpretación en los resultados al aplicarlas a sujetos de lengua nativa diferente a ésta. Otro problema que se ha mencionado es el tiempo para su aplicación. Por lo tanto, se sugirió la prueba SAHLSA-50 (Short Assessment of Health Literacy in Spanish-speaking adults), especialmente diseñada para pacientes hispano hablantes como alternativa que subsanaría los problemas antes mencionados. ${ }^{23}$ Este instrumento presenta sólidas propiedades psicométricas: el análisis factorial confirmatorio constató la unidimensionalidad de los ítems con un sólo componente con Eigenvalue $>1$; en cuanto a la validez convergente, la prueba SAHLSA logró una correlación significativa con la prueba TOFHLA en español $(r=0,65)$. De igual modo, alcanzó una alta confiabilidad interna $(\alpha$ de Cronbach $=0,92) \mathrm{y}$ una confiabilidad test-retest $(\mathrm{r}=0,86){ }^{23}$

Por lo tanto los objetivos del presente estudio se centran en identificar el porcentaje de sujetos con un adecuado conocimiento sobre la salud y determinar las variables demográficas que predicen un apropiado conocimiento sobre la misma.

\section{MATERIAL Y MÉTODOS}

La presente investigación partió de un estudio de cohorte transversal que se aplicó a 49 mujeres en edad reproductiva, de edades entre 22 y 50 años, quienes asistieron al servicio ginecológico del Hospital Dos de Mayo en Lima (Perú). Éste es un hospital de nivel primario que atiende principalmente a personas con escasos recursos económicos, y pertenece a la red de hospitales administrados por el Ministerio de Salud del Perú. Para ser incluidas en el estudio, las participantes debían ser mayores de 18 años, asistir al servicio ginecológico y tener fluidez en la lectura de textos impresos. Por el contrario, no fueron consideradas las pacientes mayores de 50 años, ni aquellas con deficiencias de visión o evidente retardo mental. Posteriormente, a cada participante se le explicó la naturaleza y el propósito del estudio y se le garantizó la confidencialidad de la información. Todas las pacientes consultadas accedieron a participar en la investigación.

Más adelante, se realizó un muestreo por conveniencia; y finalmente, en enero de 2008, se aplicó la prueba SAHLSA-50. En total, fueron dos los instrumentos que se emplearon para recopilar los datos. El primero incluía preguntas sobre variables demográficas. Inmediatamente después, cada participante completó la prueba SAHLSA-50, la cual sirve para medir el conocimiento sobre la salud. Esta prueba está compuesta por 50 ítems agrupados en dos secciones: la primera evalúa la pronunciación correcta del término y la segunda comprueba si los participantes asocian el ítem con aquel que le corresponde, para lo cual deben elegir entre tres opciones (el término correcto, un distractor y la opción "No sé”). De otra parte, para la capacitación de las encuestadoras se preparó un breve instructivo y se llevó a cabo una prueba piloto con las tarjetas que contenían los ítems de la prueba SAHLSA-50. Debido a la sencillez del formato de aplicación, tan sólo fue necesaria una sesión de preparación. Asimismo, el formato de aplicación permitió que las personas encuestadas respondieran las preguntas sin mayores dificultades, tomándoles entre seis y ocho minutos completarlas. En último lugar, se sumaron los aciertos obtenidos por cada paciente para determinar el puntaje total. El tiempo promedio para calcular dicho puntaje fue de dos a tres minutos. 


\section{Variables a medir}

Se midieron la edad, la ocupación, el estado civil y el nivel de escolaridad. El nivel de conocimiento se categorizó en dos grupos: alto conocimiento sobre la salud, cuando la encuestada obtuvo un puntaje superior al promedio, y bajo conocimiento cuando la puntuación SAHLSA-50 estuvo por debajo del promedio. Los años de escolaridad fueron definidos como el total de años de educación (secundaria o superior) que las participantes alcanzaron al momento de realizarse el estudio. La ocupación es la actividad principal, remunerada o no, que las participantes dijeron realizar. Por su parte, el estado civil tuvo cinco opciones de respuesta: soltera, casada, conviviente, divorciada y otro.

Después, se ingresaron las respuestas de las participantes a una matriz de datos SPSS, versión 12. En el análisis de datos se empleó estadística descriptiva, y para resumir la información se usaron medidas de tendencia central y dispersión en variables continuas y proporciones para variables categóricas mientras que en el análisis exploratorio se utilizó el coeficiente de correlación producto-momento de Pearson. Además, se hizo uso de la regresión lineal múltiple, empleando el método stepwise, a fin de determinar un modelo que explicara las variables predictoras del conocimiento sobre la salud. Únicamente se consideraron los coeficientes de correlación y regresión que alcanzaron significancia estadística $(\mathrm{p}<0,05)$.

\section{RESULTADOS}

El promedio de edad y de años de estudio de las 49 mujeres encuestadas fue 27,7 $\pm 8,27 \mathrm{y}$ 10,6 \pm 2,32, respectivamente. Con relación a la ocupación, $86 \%$ son amas de casa y $8 \%$ son vendedoras mientras que en lo que se refiere al estado civil, 68\% dijo ser conviviente, 18\% soltera y 14\% casada. Aunque $80 \%$ tiene algún tipo de estudios secundarios y $18 \%$ estudios superiores, $43 \%$ de las encuestadas informó no haber concluido sus estudios. La aplicación de la prueba SAHLSA-50 tomó aproximadamente 6 minutos por paciente.
En cuanto al puntaje que se obtuvo en la prueba SAHLSA-50, el rango de valores estuvo entre 22 y 50 puntos con un promedio de $37 \pm 7,15$ puntos. Dichos resultados se vieron reflejados en $43 \%$ de las encuestadas quienes lograron un puntaje inferior al promedio en la prueba de conocimiento sobre salud $(n=21)$. A partir de ésto, se puede deducir que existe una relación estadísticamente significativa entre la escolaridad, la edad y el puntaje de la prueba SAHLSA 50 (tabla 1).

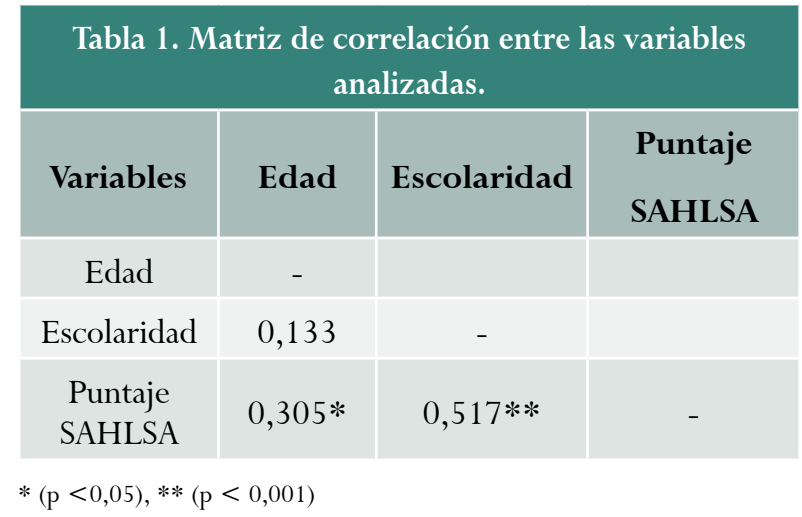

De la misma manera, el análisis de regresión lineal mostró un modelo $\left(\mathrm{R}^{2}=0,267\right)$ que descarta la edad y presenta la escolaridad como variable predictora:

Puntaje SAHLSA $=20.120+(1.596)$. Total de años estudiados + error.

De acuerdo con la ecuación obtenida:

1) Todas las entrevistadas tienen un puntaje mínimo de 20 puntos sobre 50 (37 es el punto de corte).

2) Cada incremento de 1.596 en el total de años estudiados produce un aumento de un punto en el puntaje SAHLSA-50.

Por ejemplo, si se quiere predecir el puntaje de una entrevistada que alcanzó 11 años de estudios, el cálculo sería:

Puntaje SAHLSA $=20.120+(1.596)(11)$
Puntaje SAHLSA $=20.120+17.556$.
Puntaje SAHLSA $=37.676 \rightarrow 38$ puntos $\mid$ El puntaje
real fue 40 puntos.




\section{DISCUSIÓN}

Estos resultados son importantes porque confirman las observaciones previas en cuanto a que las pacientes con menor grado de instrucción tienen menor oportunidad de entender los mensajes escritos que provienen de sus servicios de salud. Si bien es cierto que las correlaciones son moderadas teniendo en cuenta el tamaño de la muestra ( $\mathrm{n}=49)$, también es claro que ésta es una asociación importante.

Igualmente, estos hallazgos son consistentes con los estudios realizados en países anglosajones en donde la edad, el sexo y los años de escolaridad son factores asociados con el conocimiento sobre la salud, por lo que a mayor nivel de escolaridad, más profundas serán las diferencias sexuales en cuanto a éste tipo de conocimiento. ${ }^{18}$ No obstante, hay otros investigadores como Gazmararian y colaboradores quienes informan que los años de escolaridad no son predictores confiables de dicho conocimiento. ${ }^{19}$ Por medio de un estudio, cuya muestra fue de 406 mujeres entre 19 y 45 años, ${ }^{19}$ ellos encontraron que un bajo conocimiento sobre la salud estuvo asociado con pobres competencias lectoras, edad, raza y estado civil.

Ahora bien, estos datos son relevantes en la medida en que se posee una aplicación local de la encuesta SAHLSA-50 que ayuda a tener un soporte cuantitativo que permite identificar una de las causas del alto número de personas que acuden a los servicios de salud del Estado.

Por otro lado, tal como se indicó anteriormente, el entendimiento de los mensajes transmitidos a través de los medios impresos es fundamental para prevenir diferentes tipos de enfermedades, tarea que actualmente es muy importante considerando que para las ciencias de la salud en general se está fomentando la medicina preventiva.

Por el diseño empleado no es posible determinar si el bajo conocimiento sobre la salud es un problema general de la población peruana. Sin embargo, si se tiene en cuenta que se trata de un instrumento fácil de aplicar y sencillo de tabular, los investigadores que deseen trabajar con la prueba SAHLSA-50 podrían incorporarlo en proyectos de salud pública y campañas de promoción de la salud. Por consiguiente, se considera que es posible replicar el estudio en poblaciones más grandes.

De este modo, en estudios posteriores con poblaciones no urbanas cuyo idioma materno no es el español (quechua, aymara, shipibo, aguajun, etc.), se podrá comprobar si los resultados aquí presentados se mantienen.

Por otro lado, sería interesante aplicar la prueba SAHLSA-50 en adolescentes en edad escolar, dada la alta tasa de natalidad de las adolescentes y la incidencia de infecciones de transmisión sexual, así como de embarazos no deseados en estos grupos etarios. Para ello, sería deseable contar con una fuente de financiamiento que asegure la aplicación de este instrumento en estudios transversales de mayor alcance.

Tener un panorama acerca del grado de conocimiento sobre la salud por parte de los pacientes permitirá optimizar las campañas de salud, enfocándolas en determinados sectores de la población de tal forma que los planificadores de los programas de salud utilicen términos más adecuados para un mejor entendimiento o diseñen programas audiovisuales para asegurar la efectividad de estas campañas. ${ }^{24}$

En la medida en que los responsables de la elaboración de los materiales informativos de promoción de la salud tomen en cuenta los resultados de las mediciones del conocimiento sobre este tema, los pacientes reales y potenciales tomarán mejores decisiones sobre el cuidado de la misma; lo cual redundará en una mejor asignación de los recursos del Estado, el cual, a su vez, podrá dar prioridad a los grupo sociales más vulnerables. Por ello, es importante que en el diseño de las campañas de salud pública los planificadores incorporen un componente que permita medir el conocimiento sobre la salud. 


\section{CONCLUSIONES}

Los resultados muestran que las pacientes con menor grado de instrucción tendrán pocas oportunidades de entender los mensajes escritos que provengan de sus servicios de salud.

\section{REFERENCIAS}

1. Programa de las Naciones Unidas para el Desarrollo. Informe sobre el desarrollo humano 2007-2008 Lucha contra el cambio climático: solidaridad frente al mundo dividido. Alfabetización y Matriculación. New York: PNUD; 2007. p.272-74

2. Castillo-Serrano M, Sorcia-Guerra R, Jiménez-Vásquez M. Salud reproductiva, conocimientos de estudiantes en dos escuelas nivel bachillerato. Rev Enferm IMSS 2006;14:81-5.

3. Montané-Lores A, coord. ¿Cómo rinden los estudiantes peruanos en comunicación y matemática? Resultados de la evaluación nacional 2001: cuarto grado de secundaria, informe pedagógico. Lima: Ministerio de Educación; 2003.

4. Osborne H. Health Literacy from A to Z: practical ways to communicate your health message. Sudbury, Massachusetts: Jones and Bartlett; 2005.

5. Selden CR, Zorn M, Ratzan S, Parker RM, editors. Health literacy. Current bibliographies in medicine. LM Pub. No. CBM 2000-1. 2002; Bethesda, MD: National Library of Medicine. Visitado en 2009 Ago 13. Disponible en: http://www.nlm.nih.gov/ archive//20061214/pubs/cbm/hliteracy.html.

6. Chirinos J, Brindis C, Salazar V, Bardales O, Reátegui L. Perfil de las estudiantes adolescentes sexualmente activas en colegios secundarios de Lima (Perú). Rev Med Hered 1999;10:49-61.

7. Raguz M. Salud sexual y reproductiva adolescente y juvenil: condicionantes sociodemográficos e implicaciones para políticas, planes, programas e intervenciones. Lima (Perú): Centro de Investigación y Desarrollo; Instituto de Estadística e Informática; 2002.

8. Sánchez S, Atencio G, Duy N, Grande M, Flores M, Chiappe M, et al. Comparación de la educación por pares y por profesionales de la salud para mejorar el conocimiento, percepción y la conducta sexual de riesgo en adolescentes. Rev Per Med Experim Sal Púb 2003;20:206-10.

9. Sobero-García MR. Nivel de conocimiento y fuentes de información sobre métodos anticonceptivos que tienen las mujeres en edad fértil del Pueblo Joven Aeropuerto - Callao. [Tesis de Licenciatura] Lima: Facultad de Obstetricia y Enfermería, Universidad San Martín de Porres; 1994.

10. Endres LK, Sharp LK, Haney E, Dooley SL. Health literacy and pregnancy preparedness in pregestational diabetes. Diabetes Care 2004;27:331-4.

11. Fortenberry JD, McFarlane MM, Hennessy M, Bull SS, Grimley DM, St Lawrence J, et al. Relation of health literacy to gonorrhoea related care. Sex Transm Infect 2001;77:206-11.

12. Barragán M, Hicks G, Williams MV, Franco-Paredes C, Duffus W, del Rio C. Low health literacy is associated with HIV test acceptance. J Gen Intern Med 2005;20:422-5.

13. Hicks G, Barragan M, Franco-Paredes C, Williams MV, del Río C. Health literacy is a predictor of HIV/AIDS knowledge. Fam Med 2006;38:717-23.

14. Kalichman S, Ramachandran B, Katz S. Adherence to combination antiretroviral therapies in HIV patients of low health literacy. J Gen Intern Med 1999;14:267-73.

15. Safeer RS, Keenan J. Health literacy: the gap between physicians and patients. Am Fam Physician 2005;72:463-8.

16. US Department of Health and Human Services. Quick guide to health literacy; 2004. Visitado en 2009 Ago 13. Disponible en: http://www. health.gov/communication/literacy/quickguide/ factsliteracy.htm.

17. Weiss BD, Coyne C. Communicating with patients who cannot read. N Eng J Med 1997;337:272-4.

18. Aguirre AC, Ebrahim N, Shea JA. Performance of the English and Spanish S-TOFHLA among publicly insured Medicaid and Medicare patients. Patient Educ Couns 2005;56:332-9.

19. Gazmararian JA, Parker RM, Baker DW. Reading skills and family planning knowledge and practices in a lowincome managed-care population. Obstet Gynecol 1999;93:239-44.

20. Zun L, Sadoun T, Downy L. English-language competence of self-declared English-speaking hispanic patients using written tests of health literacy. J Nat Med Assoc 2006;98:912-7.

21. Baker DV, Williams MV, Parker RM, Gazmararian JA, Nurss JR. Development of a brief test to measure functional health literacy. Patient Educ Couns 1999;38:33-42. 
22. Parker RM, Williams MV, Baker DW, Nurss JR. Literacy and contraception: exploring the link. Obstet Gynecol 1996;88:72S-77S.

23. Lee SY, Bender D, Ruiz RE, Cho YI. Development of an easy-to-use Spanish health literacy test. Health Serv Res 2006;41:1392-412.
24. Romaní-Zegarra SL, Vílchez-Román C. Embarazo, hábitos de lectura y consumo de radio y televisión en adolescentes peruanas: explorando relaciones de asociación. ACIMED. 2008; 17(4). Visitado en 2009 Ago 12. Disponible en: http://scielo.sld.cu/scielo.php?script $=$ sci_arttext\& pid $=$ S1024-94352008000400008\&lng $=$ es\&nrm $=$ iso. 